Service social

\title{
Le patient cardiaque face à la transplantation
}

\section{Louise Madore et Normand Lorrain}

Volume 35, numéro 3, 1986

Les jeunes et le travail social

URI : https://id.erudit.org/iderudit/706323ar

DOI : https://doi.org/10.7202/706323ar

Aller au sommaire du numéro

Éditeur(s)

École de service social de l'Université Laval

ISSN

1708-1734 (numérique)

Découvrir la revue

Citer ce document

Madore, L. \& Lorrain, N. (1986). Le patient cardiaque face à la transplantation. Service social, 35(3), 445-456. https://doi.org/10.7202/706323ar

\section{Résumé de l'article}

Les observations sur treize candidats, dont cinq ont effectivement obtenu une transplantation cardiaque à l'Institut de cardiologie de Montréal, entre janvier 1983 et août 1984, sont regroupées en quatre phases : sélection, attente, hospitalisation et post-hospitalisation. Lors de chacune d'elles, les intervenants doivent atténuer les traumatismes psychosociaux que subissent le patient et son entourage. On analyse particulièrement les réactions des cinq personnes ayant subi la greffe et celles de leur entourage familial : besoins psycho-émotionnels, vécu personnel et interactions avec le bénéficiaire. 
MADORE, Louise, travailleuse sociale à l'Institut de cardiologie de Montréal.

LORRAIN, Normand, travailleur social à

l'Institut de cardiologie de Montréal.

\section{Le patient cardiaque face à la transplantation*}

\section{Louise Madore Normand Lorrain}

Une première expérience de greffe cardiaque a eu lieu, à l'Institut de cardiologie de Montréal, en 1968. Tenant compte des progrès scientifiques qui ont eu pour conséquence, entre autres, d'améliorer la survie, ce programme fut repris en 1982. Plusieurs facteurs ont contribué à la diminution des décès; mentionnons, entre autres: l'amélioration de la prévention et du traitement du rejet, la réduction des infections post-opératoires à court et à long terme, le diagnostic plus précoce et plus précis de la crise de rejet et, enfin, le choix plus sélectif des candidats.

Au mois d'octobre 1982, le département de chirurgie de l'Institut de cardiologie de Montréal a proposé aux travailleurs sociaux de se joindre à l'équipe multidisciplinaire de transplantation cardiaque. Ce programme de transplantation, de nature expérimentale, a exigé le développement d'un protocole qui précisait le rôle de l'intervenant et les modalités d'interventions psychosociales auprès de ce type de candidats. Il a été rédigé en tenant compte à la fois des expériences effectuées dans d'autres milieux de recherche et des aspects culturels propres à la population francophone québécoise.

Cet article résume les observations faites sur des patients ayant été acceptés pour une transplantation cardiaque. Nous les regroupons en quatre phases qui s'apparentent à celles du programme établi par le département de chirurgie de l'Institut de cardiologie de Montréal :

- une phase de sélection, où s'effectue une évaluation des dynamiques personnelle, conjugale, familiale et sociale. Cette étape décrit globalement le potentiel réactionnel à absorber l'effet d'une telle intervention chirurgicale et de ses conséquences; 
- une phase d'attente, qui se caractérise par un suivi psychosocial auprès du candidat receveur et de son entourage afin de cerner et de régulariser le niveau d'anxiété en progression ;

- une phase d'hospitalisation, comprenant une période postopératoire spécifique, où l'intervenant doit faciliter l'acceptation et l'adaptation à la greffe cardiaque et aider, s'il y a lieu, à l'intégration des changements de valeurs psychosociales;

- une phase de post-hospitalisation, où les interventions psychosociales sont orientées vers l'adaptation à un nouvel état de santé, à des capacités multipliées et à un mode de fonctionnement global. Le bénéficiaire, à cette étape, chemine vers une réinsertion sociale progressive. Il y a amélioration de son seuil de tolérance face aux difficultés, reliées à ce type d'intervention chirurgicale et à ses conséquences, qui surviennent à moyen et à long terme.

\section{Observations sur les candidats}

Les données ont été recueillies sur une période de dix-huit mois, de janvier 1983 à août 1984. Treize candidats ont été évalués: trois femmes et dix hommes.

Les caractéristiques des femmes sont les suivantes : elles ont entre 21 ans et 45 ans; une est célibataire et deux sont mariées ; elles résident majoritairement hors de la région métropolitaine de Montréal ; deux d'entre elles ont une scolarité de niveau secondaire et l'autre, de niveau collégial; au plan du travail, l'une est secrétaire et les deux autres ne travaillent pas à l'extérieur ; il est à remarquer qu'une des candidates décédera durant la période d'attente, tandis que les deux autres subiront l'intervention chirurgicale.

Chez les hommes, l'âge varie entre 33 ans et 52 ans et une majorité sont dans la quarantaine. Six d'entre eux sont mariés, trois vivent en union libre et un est célibataire. La majorité des candidats demeurent à Montréal alors que trois vivent dans d'autres régions du Québec. Au plan de la scolarité, deux ont un niveau primaire, quatre secondaire, deux collégial et deux un niveau universitaire. Trois candidats sont des commerçants de services ou de produits et les autres s'identifient comme professionnel, administrateur intermédiaire, employé de bureau, contremaître, plombier, retraité, et invalide. Des dix candidatures à la transplantation cardiaque, quatre furent refusées pour des raisons médicales; trois candidats sont décédés en période d'attente et trois ont finalement subi l'intervention. Bref, sur une période de dix-huit mois, deux femmes et trois hommes ont obtenu une greffe cardiaque. 


\section{Adaptation et réadaptation}

Nous définissons l'adaptation par la capacité, pour chacun, de traverser une ou des étapes données, dans un contexte circonscrit. Les principaux éléments retenus pour l'évaluer sont : la réaction au degré d'évolution de l'état physique, la nature et quantité d'activités ainsi que la qualité des relations psychosociales. Par ailleurs, la réadaptation se manifeste par les résultats obtenus lors de la reprise ou du développement d'un mode de vie. Dans cette étude, le processus de réadaptation, composé de divers éléments d'adaptation, débute dès le moment où les clients prennent conscience de la gravité de leur maladie cardiaque et des limites du traitement médical, perçu comme un élément de maintien à brève échéance.

Tout au long des étapes du programme, nous observerons la qualité et l'évolution de l'adaptation du candidat, de son entourage et des ressources offertes aux plans médical et paramédical. À la fin de la période d'observation, il s'avérera que les cinq personnes soumises à une transplantation cardiaque présentent des éléments d'adaptation favorables à leur processus de réadaptation.

Voici comment s'est effectué le cheminement du bénéficiaire et de son entourage vers la réadaptation: les observations qui suivent sont situées à l'intérieur des différentes phases définies par le programme de transplantation cardiaque.

\section{Phase de sélection}

L'annonce de la possibilité d'une transplantation cardiaque entraine, chez le bénéficiaire, certaines réactions émotionnelles qui se traduisent par une prise de conscience de la gravité de son état cardiaque, de ses limites, de la notion de la mort et des difficultés de survie qui sont associées à une telle intervention. L'émotion dominante ressentie est l'insécurité, et le bénéficiaire constate qu'il n'a pas le choix de refuser ou d'accepter la proposition d'une chirurgie et de franchir les étapes qui confirmeront les possibilités de l'intervention.

L'entourage partage les mêmes inquiétudes concernant la gravité de la maladie et les possibilités de réussite ou d'échec d'une transplantation cardiaque. Il craint aussi, advenant que l'intervention soit impossible, les réactions du candidat. Seront-ils capables de lui offrir le support adéquat tout en sachant qu'il y aura mort à brève échéance?

Les personnels médical et social sont présents auprès du bénéficiaire et de son entourage familial lorsque la demande d'intervention est 
soumise à l'évaluation. Les interventions psychosociales visent alors à canaliser les énergies nécessaires qui permettront, soit d'affronter un rejet de la candidature, soit de poursuivre les étapes qui mèneront à l'intervention et à la réadaptation.

\section{Phase d'attente}

Lorsque le candidat est accepté, l'insécurité fait place, en partie, à l'espoir : espoir de la réussite de l'intervention, espoir de survie. Par contre, plus le délai se prolonge, plus l'insécurité augmente et, par conséquent, le besoin d'être rassuré. Les interrogations initiales quant aux possibilités de survie refont surface et le bénéficiaire se demande s'il vivra jusqu'au moment de la greffe ; ces inquiétudes provoquent de l'angoisse et un désintérêt progressif à l'égard des activités quotidiennes. Tout est désormais centré et organisé en fonction de l'appel éventuel qui confirmera la possibilité de la greffe cardiaque. Lors de cette période d'attente, le candidat se préoccupe donc généralement peu du contenu du programme et de ses conséquences. Sa seule priorité est d'obtenir l'intervention.

Au cours de cette période, le niveau de tension de l'entourage est variable. On remarque que les personnes significatives, qui ont et qui maintiennent des activités hors foyer, parviennent davantage à maîtriser cette anxiété. Par contre, celles qui vivent continuellement auprès du candidat sont soumises, elles aussi, à beaucoup de tension.

L'augmentation du délai entre l'évaluation et l'intervention et, par conséquent, de l'inquiétude du bénéficiaire et de son entourage, accroît le besoin de ressources psychosociales. Durant cette période, les intervenants psychosociaux évaluent s'il y a des problèmes de communication et si ceux-ci contribuent à augmenter l'anxiété. Ils tentent également de cerner les perturbations de l'affect, telles la peur, l'insécurité et l'angoisse, qui occasionnent des attitudes et des comportements excessifs. Les travailleurs sociaux cherchent enfin à faciliter l'évolution adéquate des mécanismes de défense tout en dédramatisant la situation : situer l'intervention dans un cadre réaliste permet en effet, au candidat et à son entourage, de maintenir l'équilibre psychoémotionnel et social nécessaire pour prévenir un état dépressif aigu. 


\section{Phase d'hospitalisation}

\section{Période pré-opératoire}

La convocation du candidat pour son admission en vue d'une greffe cardiaque provoque un choc émotionnel. Généralement, le bénéficiaire parvient à rationaliser cette crainte et on peut supposer que les interventions réalisées durant la période d'attente ne sont pas étrangères à cette réaction. Il a maintenu ou développé des mécanismes de défense et une certaine capacité d'organisation psycho-émotionnelle. Lors de l'hospitalisation, de façon générale, le candidat à la greffe et ses proches vivent des liens affectifs très étroits et significatifs, imprégnés de support et d'espoir. Au moment de l'admission et au cours de la période opératoire, les ressources psychosociales sont mises à la disposition du candidat et de son entourage. La disponibilité de ces ressources rassure le bénéficiaire et permet à l'entourage d'exprimer et de supporter l'intensité de l'inquiétude provoquée par ce type de chirurgie.

\section{Période post-opératoire}

\section{a) Isolement}

Lors du séjour à la chambre de type incubateur, l'ensemble des malades réagissent positivement ; certains apporteront même avec eux un objet personnel, comme pour concrétiser le fait qu'ils sont bien vivants. Ce comportement semble contribuer au maintien de leur motivation et favorise l'évolution de sentiments et d'attitudes positifs, ce qui constitue un atout pour le processus de récupération physique. Un élément retient cependant notre attention : la majorité des bénéficiaires ont une réaction euphorique à la suite de l'opération. Il semble que le fait d'avoir obtenu la greffe cardiaque tant souhaitée atténue les inquiétudes antérieures et provoque cette attitude. Par contre, au fur et à mesure de leur implication dans le programme et de la prise de conscience des conséquences possibles, d'autres types de craintes s'installent, tels que la possibilité d'un rejet, l'avènement de complications qui pourraient amener une augmentation ou une modification des médicaments, l'apparition d'effets secondaires, etc. Ces nouvelles sources d'insécurité sont parfois alimentées par les nombreuses précautions que prend l'équipe médicale. Ceci provoque même, à l'occasion, une réticence à quitter la chambre pour subir des examens médicaux (par exemple: crainte d'infection). 
Une fois la greffe réalisée, la réaction des proches en est une de soulagement et de répit. Il se crée un climat de confiance avec l'équipe médicale, ce qui facilite la collaboration et la compréhension de l'entourage face à l'organisation des soins prévue pour le bénéficiaire.

En général, les services psychosociaux qui sont offerts, à ce stade, sont intensifs et visent à favoriser le cheminement et l'adaptation du bénéficiaire et de son entourage. Lors de l'hospitalisation, le malade partage plus facilement son vécu, et le contenu des entrevues varie selon les événements quotidiens : informations reçues par le bénéficiaire ou sa famille, problèmes spécifiques, de santé ou autres, etc. Les interventions psychosociales se focalisent principalement sur ce vécu et les états affectifs qui en découlent. On remarque, toutefois, que les proches du bénéficiaire font une utilisation variable des ressources mises à leur disposition; leur perception de la situation et l'évaluation de leurs propres capacités d'adaptation influencent ce recours aux services qui leur sont offerts.

\section{b) Intégration}

La période d'intégration se caractérise par un allégement des interventions médicales, ce qui accorde au bénéficiaire, entre autres, plus de mobilité à l'extérieur de sa chambre. On observe, à ce moment, une hausse de confiance chez la personne greffée, qui est consciente d'avoir surmonté le défi ; même si elle demeure confrontée au phénomène du rejet cardiaque, sa réaction aux médicaments qui contrôlent le rejet la rassure et lui confirme que son organisme va s'adapter. De façon générale, cette période positive s'accompagne de changements d'attitudes et de comportements: le bénéficiaire développe un sentiment de gratitude face au personnel hospitalier et il démontre de l'intérêt et de la disponibilité face aux exigences du programme ; en ce qui concerne ses proches, ils s'impliquent davantage et tendent à reprendre leurs rôles sociaux (planification, prises de décision, etc.). Au cours de cette période d'hospitalisation, les interventions portent principalement sur les réactions au vécu hospitalier et sur les réactions au programme de transplantation cardiaque.

On remarque chez la personne greffée un besoin d'attention, la surprise de ne plus souffrir et une satisfaction d'avoir retrouvé une certaine capacité physique. Quelques-unes d'entre elles manifestent même de la curiosité par rapport aux caractéristiques du donneur : âge, sexe, cause de décès, etc.

Les modifications des conditions de l'hospitalisation, à cette étape, ainsi que la réponse rapide des intervenants médicaux et psychosociaux 
aux interrogations et aux inquiétudes du bénéficiaire, favorisent un état de sécurité. Cependant, certains patients peuvent développer une dépendance face à l'hôpital; il faut alors les préparer au retour à domicile en favorisant l'autonomie, en les habilitant à prendre seuls la médication recommandée, en intervenant auprès des proches pour les aider à comprendre le bénéficiaire et la maladie, et pour les inciter à adopter un comportement qui n'instaure ni ne maintienne un phénomène de dépendance.

Toute l'importance de l'intervention médicale se manifeste au moment où la personne greffée réalise qu'elle devra prendre régulièrement des médicaments et lorsqu'elle prend conscience de leurs effets secondaires. Elle saisit graduellement la portée des implications et des conséquences de la transplantation. À ce stade, il arrive que le greffé cardiaque se perçoive comme collaborateur actif à la réussite du programme ; il s'ensuit un vedettariat qui s'estompe progressivement.

Lors de la période d'intégration, les membres de l'entourage observent l'autonomie physique du greffé et ses interactions avec l'équipe soignante. Ils portent également une attention particulière aux attitudes et aux réactions émotives ainsi qu'aux types de communication que le bénéficiaire établit avec eux. Ils partagent son enthousiasme relativement à l'amélioration globale de son état. Toutefois, l'exagération au plan des sentiments (angoisse ou euphorie) et la difficulté à s'adapter aux recommandations du programme (par exemple, à l'apprentissage de la prise des médicaments), inquiètent les parents qui tendent alors à manifester une certaine surprotection.

Le suivi médical et psychosocial, à cette étape, vise à dédramatiser toute nouvelle crainte (effets secondaires dus aux médicaments, rejet, etc.) et à rassurer le bénéficiaire sur son intégration à l'environnement familial et social. Les intervenants lui font prendre conscience des progrès obtenus, indicateurs du succès de l'intervention. En général, la motivation et la collaboration entre le bénéficiaire et le travailleur social sont maintenues. Par contre, en ce qui concerne l'entourage, on remarque que seuls les membres de la famille qui anticipent une difficulté reliée au type de personnalité de la personne greffée ou à la qualité des interactions antérieures ou actuelles, continuent un suivi psychosocial.

\section{Phase post-hospitalisation}

Lors de la phase de post-hospitalisation, l'intervention auprès du bénéficiaire touche les aspects suivants: les effets secondaires des 
médicaments, le contrôle médical, et la réinsertion sociale. L'intervention auprès de l'entourage porte surtout sur ses réactions à la réinsertion du bénéficiaire.

\section{Effets secondaires}

Les effets secondaires des médicaments sont multiples; ceux qui apparaissent le plus fréquemment, sont : les infections, les changements corporels, les malaises physiques et les modifications du comportement. Les capacités d'adaptation aux médicaments, d'acceptation de leurs effets secondaires et les réactions de l'environnement à ces effets varient dans chaque cas. Certains problèmes physiques amènent des réactions psychologiques importantes; mentionnons, entre autres, les modifications corporelles au plan du faciès, et les infections (herpès, infection vaginale, etc.). Malgré les difficultés d'adaptation, il apparaît, cependant, que le fait de survivre à une greffe cardiaque soit plus important que ces changements. La réussite de la transplantation cardiaque restreint l'impact affectif qui pourrait résulter de ces modifications physiques. Seul le rejet massif provoque une perturbation émotionnelle importante, d'une part parce qu'il menace la survie du bénéficiaire et, d'autre part, parce qu'il entraîne la prise de nouveaux médicaments qui peuvent provoquer d'autres effets secondaires non connus.

\section{Contrôle médical}

La totalité des bénéficiaires, et même ceux qui résident loin de I'Institut, observent le contrôle médical (examens de contrôle et rendezvous médicaux en clinique externe) prévus par le programme. En ce qui a trait aux biopsies, la motivation des patients varie selon l'expérience qu'ils ont de ce type d'examen, leur degré d'adaptation aux divers résultats obtenus, l'importance qu'ils y accordent et leur propre perception de leur état physique.

Les rencontres en clinique externe entre le cardiologue, le bénéficiaire et l'entourage les rassurent généralement au plan de l'évolution physique. Ces échanges peuvent également permettre de dépister des problèmes d'interrelations vécus dans le milieu familial et de prévenir la cristallisation des difficultés. 


\section{Réinsertion sociale}

Au plan de la réinsertion sociale, les intervenants se préoccupent de la qualité des échanges entre la personne greffée et son entourage, tant au niveau des communications qu'au niveau affectif, ainsi que des rôles, des besoins et des attentes de chacun. Ils tiennent compte, aussi, de la participation aux activités sociales telles que le travail et les loisirs, que celles-ci soient nouvelles ou antérieures à la transplantation.

\section{Aspects psycho-émotionnels}

La condition sociale, l'âge, les réactions du bénéficiaire et de l'entourage face aux nouvelles capacités de la personne qui a subi l'intervention, ainsi que l'enthousiasme et le dynamisme qui peuvent en découler, sont des éléments qui influencent le type de besoins psychosociaux. Le patient jeune et dépendant du réseau familial accepte et trouve normale la protection qu'on lui offre à son retour à la maison. Par contre, celui qui a un conjoint se sent plus en sécurité et s'oppose plus facilement à une situation de surprotection. Le retour à la vie normale semble plus rapide lorsque supporté par la stabilité d'une relation socio-affective. On remarque, de plus, que les bénéficiaires effectuent, au cours des différentes phases du programme, plusieurs modifications d'attitudes, de comportements et de valeurs par rapport à la vie.

Les membres de l'entourage ne présentent pas de telles modifications d'attitudes et de valeurs. Lorsque le bénéficiaire revient à domicile, ils ne sont pas toujours d'accord avec sa conception de la réadaptation physique et du rythme qu'il doit accorder à la reprise de ses activités. Ils sont étonnés par l'attitude psychologique générale de la personne greffée, qui semble plutôt centrée sur elle-même. Ainsi, les membres de la famille s'attendent à une période de convalescence basée sur des restrictions et à un retour progressif aux activités. Ils sont toutefois confrontés à un individu qui démontre un dynamisme et une capacité physique plus élevés que prévu. Cela entraîne inévitablement des difficultés de communication, des modifications dans les relations affectives et des changements psychosociaux accentués par les effets secondaires des médicaments. Ils se sentent bousculés car ils doivent admettre que le bénéficiaire manifeste un état de santé amélioré et des transformations psychologiques et sociales relativement à son mode de vie et à ses valeurs personnelles.

Au plan de l'affect, toutefois, la restructuration n'est pas aussi évidente car la personne greffée vit un traumatisme relié à la maladie 
cardiaque, à l'hospitalisation et au traitement médical. Alors que le bénéficiaire cherche à réintégrer ses rôles et ses fonctions sociales, on note certaines perturbations de l'affect qui sèment le doute sur la possibilité de reprendre les modes de fonctionnement conjugal et familial antérieurs. Le bénéficiaire et sa famille doivent s'ajuster en tentant de comprendre les modifications qui se sont opérées et leur divergence de conception du vécu actuel.

\section{Aspect social}

Parmi les cinq personnes greffées, un homme et une femme sont parvenus à un retour complet à la vie normale, c'est-à-dire qu'ils occupent un travail rémunéré ou ont repris les activités quotidiennes et sociales qu'ils avaient avant la maladie ou en ont développé de nouvelles. Les trois autres patients greffés ne sont pas revenus à une vie normale pour différentes raisons : au cours des étapes de réadaptation, l'un des bénéficiaires a éprouvé des difficultés d'intégration au travail ; le second, en dépit d'un niveau élevé de motivation nécessaire à la reprise des activités sociales, a été gêné par des problèmes physiques; enfin, le troisième a été confronté à un environnement familial réticent qui a difficilement accepté la nature de l'intervention chirurgicale, ses conséquences et les exigences rattachées à la survie et, de plus, il n'a pu reprendre son emploi antérieur à cause des conditions de travail.

De façon générale, le bénéficiaire prévoit peu de difficultés pour le retour à la vie normale et l'entourage entend recourir à ses propres ressources psychosociales. Tous deux maintiennent une collaboration au plan du suivi médical, conscients de l'importance de la dimension physiologique et de leur impuissance à ce niveau. Lorsque les résultats médicaux positifs s'accumulent, la motivation au suivi médical et paramédical diminue chez la majorité d'entre eux. Lors du retour à domicile, la fréquence des rencontres psychosociales varie selon les désirs de l'entourage et de la personne greffée. Dans l'ensemble, ceux-ci se considèrent aptes à répondre à leurs besoins et attentes mutuelles avec leurs propres ressources et celles de leur environnement familial et social. Par contre, ils désirent reprendre les consultations psychosociales régulières lorsqu'il n'y a pas d'amélioration au plan de la réadaptation conjugale, familiale et sociale et lorsque certaines situations, reliées à leur processus de réadaptation, deviennent problématiques. 


\section{Conclusion}

Cette étude, réalisée de janvier 1983 à août 1984, nous permet de faire ressortir les éléments suivants :

- les phases de sélection et d'attente sont caractérisées par un traumatisme majeur, au plan psycho-socio-émotionnel, pour les candidats et leur entourage familial. La nature du choc émotionnel provient des éléments déclenchants qui sont, pour la majorité, le désespoir face à la gravité de la cardiopathie, l'insécurité et l'espoir d'obtenir une greffe cardiaque. Les interventions des personnels médical et psychosocial portent sur l'adaptation à la période d'attente, la préparation du candidat et de son entourage à l'éventualité d'un décès et la préparation à la chirurgie. Lorsque le bénéficiaire et l'entourage ont franchi l'étape de l'évaluation, on remarque qu'ils sont motivés et qu'ils collaborent à un suivi psychosocial et médical structuré ;

- au début de la phase d'hospitalisation, le bénéficiaire vit une période d'isolement où l'on observe entre autres un affect fragile ;

- après la transplantation, le bénéficiaire et son entourage doivent apprendre les exigences du programme ; cette situation nécessite la concertation des ressources médicales et paramédicales afin de faciliter l'acceptation et l'adaptation aux nouveaux éléments qui s'insèrent dans le vécu quotidien ;

- le retour à domicile se caractérise par une crise au plan des rôles, et par des malaises au niveau des communications et des relations affectives; des perceptions d'orientations différentes du mode de vie en sont à l'origine. En somme, on constate que le bénéficiaire désire reprendre un ensemble d'activités et qu'il exprime de nouvelles prises de position. Par contre, l'entourage présente une réaction de surprise et paraît démuni, car il s'attendait plutôt à la reprise d'un mode de vie identique à celui vécu antérieurement à la maladie. Ainsi, il y a obligation de réaménager et de clarifier les besoins et les attentes de chacun par rapport à leur intégration et leur implication dans l'organisation de leur vie future.

Les modes d'intervention s'adaptent aux conditions et situations présentées par les futurs candidats et par les personnes greffées : le suivi psychosocial est d'ordre individuel, conjugal et parfois familial, et les modèles d'intervention privilégiés s'apparentent aux différentes phases du programme et tiennent compte des aspects individuels et environnementaux. 
Les résultats de cette recherche nous ont permis d'identifier deux objectifs qu'il faudrait désormais chercher à atteindre :

- le développement d'un mécanisme qui aiderait l'entourage à entreprendre un processus d'évolution psycho-socioémotionnelle à l'intérieur du programme, et qui tiendrait compte de ses caractéristiques propres face à la greffe cardiaque. Ceci favoriserait la compréhension de l'ensemble des transformations d'attitudes et de comportements qui sont observées chez la personne greffée et l'entourage familial, dans les différentes phases du programme, et permettrait d'envisager une réadaptation réaliste pour chacun ;

- l'observation systématique et l'analyse des structures et des organismes sociaux quant à leurs attitudes face aux personnes greffées et quant à leur perception de leur intégration globale et de leur rôle économique. La réalisation de cet objectif implique, entre autres, l'employeur, l'assureur et le législateur. À ce niveau, I'Institut de cardiologie de Montréal est en mesure d'assumer un rôle de prévention et d'éducation sociale, et devra instaurer des mécanismes pour répondre à ces nouveaux besoins.

\section{Note}

* Les auteurs remercient les $D^{r}$ Marcel Boulanger, directeur des services professionnels, et le Dr L. Conrad Pelletier, F.R.C.F. (C), chef du département de chirurgie, de l'Institut de cardiologie de Montréal, pour leur collaboration. 